\title{
A Bilateral Advantage for Storage in Visual Working Memory
}

\author{
Akina Umemoto, Trafton Drew, Edward F. Ester, and Edward Awh \\ University of Oregon
}

\begin{abstract}
Various studies have demonstrated enhanced visual processing when information is presented across both visual hemifields rather than in a single hemifield (the bilateral advantage). For example, reported that observers were able to track twice as many moving visual stimuli when the tracked items were presented bilaterally rather than unilaterally, suggesting that independent resources enable tracking in the two visual fields. Motivated by similarities in the apparent capacity and neural substrates that mediate tracking and visual working memory (WM), the present work examined whether or not a bilateral advantage also arises during storage in visual WM. Using a recall procedure to assess working memory for orientation information, we found a reliable bilateral advantage; recall error was smaller with bilateral sample displays than with unilateral displays. To demonstrate that the bilateral advantage influenced storage per se rather than just encoding efficiency, we replicated the observed bilateral advantage using sequentially presented stimuli. Finally, to further characterize how bilateral presentations enhanced storage in working memory, we measured both the number and the resolution of the stored items and found that bilateral presentations lead to an increased probability of storage, rather than enhanced mnemonic resolution. Thus, the bilateral advantage extends beyond the initial selection and encoding of visual information to influence online maintenance in visual working memory.
\end{abstract}

\begin{abstract}
The organization of the visual system is primarily contralateral such that information from the left visual hemifield is initially processed in the right hemisphere while information from the right visual hemifield is processed in the left hemisphere. Although information from these separate pathways is eventually integrated via the connecting fibers of the corpus callosum, various studies have reported enhanced performance when items are distributed across both hemifields such that both the right and left hemispheres receive the initial input, compared to when a single hemisphere processes the same amount of information. This effect has been termed the bilateral advantage. Alvarez and Cavanagh (2005) provided one of the most compelling demonstrations of a bilateral advantage using a task that required observers to simultaneously track multiple targets that were presented in either unilateral or bilateral displays. This study revealed an approximate doubling of tracking capacity when the targets were spread across both hemifields compared to when the same number of targets occupied a single hemifield, suggesting independent attentional capacities in the right and left cerebral hemispheres. The Alvarez and Cavanagh (2005) findings dovetail with several other studies that also showed a clear bilateral advantage during encoding-limited tasks that required pattern matching (Muller, Malinowski, Gruber and Hillyard, 2003; Reuter-Lorenz, Stanczak and Miller, 1999; Sereno and Kosslyn, 1991) or rapid target discrimination (Awh and Pashler,
\end{abstract}

(C) 2010 Elsevier B.V. All rights reserved.

Correspondence: Edward Awh 1227 University of Oregon Eugene, OR 97403 awh@ uoregon.edu.

Publisher's Disclaimer: This is a PDF file of an unedited manuscript that has been accepted for publication. As a service to our customers we are providing this early version of the manuscript. The manuscript will undergo copyediting, typesetting, and review of the resulting proof before it is published in its final citable form. Please note that during the production process errors may be discovered which could affect the content, and all legal disclaimers that apply to the journal pertain. 
2000; Carlson, Alvarez, and Cavanagh, 2007; Kraft, Muller, Hagendorf, Schira, Dick, Fendrich, and Brandt, 2005; Kraft, Pape, Hagendorf, Schmidt, Naito and Brandt, 2007; Liu, Jiang, Sun and He, 2009; Scalf, Banich, Kramer, Narechania, and Simon, 2007).

Although the tracking task employed by Alvarez and Cavanagh (2005) differed in important ways from the tasks used in other demonstrations of the bilateral advantage, they offered a hypothesis that is consistent with the full range of findings. Specifically, Alvarez and Cavanagh (2005) suggested that there may be hemisphere-specific resources that are required for the initial selection of target items, while later stages of processing such as identification and memory storage may not show hemifield independence. With the exception of split-brain patients, this hypothesis was in line with previous failures to find strong evidence of hemisphere-dependent resources in normal, healthy individuals in visual search tasks (Luck, Hillyard, Mangun, and Gazzaniga, 1989) and memory storage (Duncan, Bundesen, Olson, Humphreys, Chavda and Shibuya, 1999). Thus, while Alvarez and Cavanagh (2005) provided definitive evidence of a bilateral advantage during attentive tracking, similar evidence from memory-limited tasks has been elusive (but see Delvenne, 2005). Nevertheless, there is a growing body of neural and behavioral evidence suggesting functional overlap between attentive tracking and storage in visual WM. Past studies have reported a similar capacity limit for the two such that approximately 3-4 objects can be actively maintained in visual working memory (Luck and Vogel, 1997; Pashler, 1988; Sperling, 1960; Vogel, Woodman \& Luck, 2001), and tracked simultaneously (Cavanagh and Alvarez, 2005; Oksama and Hyönä, 2004; Pylyshyn and Storm 1988). In line with these similar capacity limits, Oksama and Hyönä (2004) found reliable correlations between an individual's performance in MOT and visuospatial WM tasks. Likewise, Fougnie and Marois (2006) demonstrated strong dual task interference effects when subjects were required to simultaneously track objects and store items in visual WM. Finally, fMRI and ERP studies have suggested that similar neural regions mediate attentive tracking and storage in visual WM. Functional MRI studies have revealed common brain regions that are active during both MOT and VSTM tasks, including the frontal eye fields and intraparietal sulcus (Culham, Brandt, Cavanagh, Kanwisher, Dale \& Tootell, 1998; Culham, Cavanagh \& Kanwisher, 2001; Howe, Horowitz, Morocz, Wolfe and Livingstone 2009; Jovicich, Peters, Koch, Braun, Chang \& Ernst, 2001; Linden, Bittner, Muckli, Waltz, Kriegeskorte, Goebel, Singer \& Munk, 2003; Todd and Marois, 2004, 2005; $\mathrm{Xu}$ and Chun, 2006). More recently, Drew and Vogel (2008) recorded event-related potentials during an MOT task, and found a robust CDA (contralateral delay activity) waveform that strongly predicted individual tracking ability. The CDA waveform, whose activity is likely to arise from the intraparietal sulcus (IPS), has been previously shown to be a robust predictor of capacity in visual WM (Vogel and Machizawa, 2004; Vogel, McCollough, and Machizawa, 2005). A similar link between WM capacity and activity in the posterior parietal cortex was also observed by Todd and Marois (2005) in a voxelwise fMRI analysis. These results together suggest that a common neural resource may mediate performance both in the tracking and visual WM tasks, and highlight the possibility that a bilateral advantage may influence performance in both tasks.

Indeed, there is one published demonstration of a bilateral advantage during a visual working memory task. Using a standard change detection task, Delvenne (2005) measured capacity in a spatial WM task and found a reliable enhancement of capacity when the stored positions were presented bilaterally relative to when they were presented unilaterally. This result is consistent with the hypothesis that WM storage is improved in the bilateral condition, but one alternative explanation requires consideration. Given that success in the change detection task is dependent on both successful encoding and storage of the target items, the possibility remains that the bilateral advantage in the Delvenne (2005) study resulted from differences in the quality of stimulus encoding in the bilateral and unilateral conditions. Thus, a key goal of the present research was to re-examine whether WM storage is subject to a bilateral advantage while 
attempting to rule out stimulus encoding as the source of this putative effect. Conclusive evidence of a bilateral advantage during WM storage would show that even relatively late stage memory processes are influenced by hemisphere-specific resource limits. To anticipate the results, our studies showed a reliable bilateral advantage during the maintenance of orientation information in visual WM. Furthermore, this bilateral advantage was robust even when the memoranda were presented sequentially, thereby precluding the possibility of a bilateral advantage during stimulus encoding. These data complement those of Delvenne (2005) by showing that storage in WM per se is enhanced when the stored items are initially presented to separate hemispheres.

Finally, we examined which aspect of memory storage was affected by the bilateral advantage. Recent evidence has suggested that WM capacity may be determined by two distinct aspects of memory ability, such that separate factors determine the maximum number of items that can be held in WM, and the precision or resolution of those memory representations (Awh, Barton, and Vogel, 2007; Barton, Ester, and Awh, 2009; Scolari, Vogel and Awh, 2008; Xu and Chun, 2006; Zhang \& Luck, 2008). Xu and Chun (2006) provided neural evidence for this dissociation by demonstrating that activity in distinct neural regions reflected the number of items stored in working memory, and the complexity of the stored items. Given that higher resolution is needed for accurate performance with complex objects, the findings of Xu and Chun (2006) suggest that distinct neural regions mediate number and resolution in visual WM. In line with this neural dissociation, an analysis of individual differences (Awh et al., 2007) found no correlation between these two aspects of memory storage. That is, subjects who could hold a larger number of items in WM were not necessarily the same subjects who could maintain the items with higher resolution. This raises an interesting question about how bilateral presentations may affect storage in working memory. When the items to be stored are bilaterally arrayed, does this enable the storage of a larger number of items, or does it instead influence the resolution of the stored representations? To answer this question, we employed an analytic procedure developed by Zhang and Luck (2008) that enables separate estimates of the number and resolution of the representations stored in WM. This procedure replicated our findings of a reliable memory advantage in the bilateral condition. Importantly, the bilateral advantage was found only with respect to the number of items maintained in WM; the resolution of the stored representations was equivalent in the bilateral and unilateral conditions.

\section{Experiments 1a \& 1b}

We used an orientation recall task to test whether visual WM shows a bilateral advantage.

Subjects in Experiment 1a were briefly presented with two teardrop stimuli whose orientations were randomly selected from 72 possible orientation angles with 5 degree increments from 0 to 360 degrees. In Experiment 1b, the possible orientations were spaced more finely (in one degree increments) to allow a more precise measurement of orientation precision. These stimuli were presented either within or between the two hemifields, and performance was measured by recording the angular separation between the subjects' response and the probed sample item. Finally, one central goal of the present research was to test whether the bilateral advantage leads to enhanced storage per se rather than enhanced encoding of the sample stimuli. After all, most previous demonstrations of the bilateral advantage are from strongly encoding limited tasks (e.g., Awh and Pashler, 2000; Kraft, Muller, Hagendorf, Schira, Dick, Fendrich, and Brandt, 2005; Kraft, Pape, Hagendorf, Schmidt, Naito and Brandt, 2007; Reuter-Lorenz, Stanczak and Miller, 1999; Sereno and Kosslyn, 1991; Scalf, Banich, Kramer, Narechania, and Simon, 2007). Thus, in both Experiment 1a and 1b, stimuli were presented either all at the same time (simultaneous condition), or one at a time in sequence (sequential condition). The rationale was that sequential encoding would rule out any possible bilateral advantage during encoding because for both the bilateral and unilateral conditions, the sample stimuli were encoded in only one hemifield at a time. Thus, a bilateral advantage during the sequential 
condition could not be explained by differences in encoding quality. We included both the simultaneous and sequential conditions to enable a direct comparison of the size of the bilateral advantage in these two conditions.

\section{Method}

Subjects

Two groups of subjects from the University of Oregon community (14 for Experiment 1a, and 30 for Experiment 1b) participated in a one hour experimental session for either psychology course-credit or for monetary compensation. All subjects reported normal or corrected-tonormal vision.

\section{Stimuli}

Experiment 1a-The stimuli were "teardrops" that had a length of $2.1^{\circ}$ and a width of $1.3^{\circ}$ (see Figure 1). The orientation of each stimulus was randomly selected from 72 possible angles that started at $0^{\circ}$ and were evenly spaced in $5^{\circ}$ increments around the full 360 degree space. There stimuli were black, presented on a gray background. The two stimuli appeared $4.1^{\circ}$ from the central fixation. The stimuli were presented either within the same hemifield (vertically aligned) or across the two hemifields (horizontally aligned). The center-to-center distance between each item was $4.9^{\circ}$ for the vertical, and $5.7^{\circ}$ for the horizontal alignment (this distance was equated in Experiment 1b). The simultaneous condition contained two stimuli appearing either across the two hemifields or within the same hemifield for $150 \mathrm{~ms}$, whereas one item was presented at a time for $150 \mathrm{~ms}$ each with a $300 \mathrm{~ms}$ inter-stimulus interval (this made the total duration of each trial, excluding the latency of subjects' response, longer in the sequential condition than in the simultaneous condition ( $2150 \mathrm{~ms}$ vs. $2600 \mathrm{~ms})$ ). The location of the second item in the sample array in the sequential condition was equally likely to fall in the same hemifield as in the opposite hemifield, hence the position of the first items in the sequential condition provided no information regarding whether the subsequent stimuli would be in the same or opposite hemifield. The next trial began 1 second after subjects selected a target orientation and pressed the "enter" key. All subjects participated in both the simultaneous and sequential encoding conditions (blocked), and the order of conditions was counterbalanced across subjects.

Experiment 1b-In Experiment 1b, two changes were made. First, the stimulus positions were adjusted. In Experiments 1a, the center-to-center distance between objects was slightly larger for the bilateral displays than for the unilateral displays (by $0.7^{\circ}$ ). Although the distance between objects in the unilateral displays was well beyond the range of critical spacing in Experiments 1a, arguing against differential crowding effects in the bilateral and unilateral conditions, inter-stimulus distances were equated in the bilateral and unilateral conditions of Experiments $1 \mathrm{~b}$ (and Experiment 2). Second, the possible stimulus angles varied in 1 degree increments across the entire $360^{\circ}$ space. The stimuli were outlined circles with an oriented bar that was stretched from its center dot (see Figure 2). Each stimulus was $6.1^{\circ}$ in diameter with a center-to-center distance of $6.8^{\circ}$, and $9.7^{\circ}$ from the central fixation. In the test array, one of the stimuli reappeared without its oriented bar (i.e., the outlined circle with a center dot).

Subjects used the computer mouse to click on the tested item to indicate the orientation of the bar for the item that was in the same position in the sample array. The next trial began immediately after the mouse click that indicated the subjects' response.

\section{Procedure}

Experiment 1a-Each trial began with the onset of a fixation point for one second (see Figure 1). Immediately afterwards, a sample array containing two teardrop stimuli in either a single hemifield or across both hemifields was presented for $150 \mathrm{~ms}$ for the simultaneous condition, 
while the sequential condition showed one teardrop at a time for $150 \mathrm{~ms}$ each with a $300 \mathrm{~ms}$ of inter stimuli interval (this procedure made the total trial duration longer for the sequential condition by $450 \mathrm{~ms}$ ). Following the offset of the sample array, there was a $1 \mathrm{~s}$ delay period, after which a test array containing a single randomly oriented teardrop was presented. Subjects adjusted the orientation of the item in the test array to match the orientation of the item from the same position in the sample array. The up and down arrow keys guided subjects through 72 possible stimulus orientations (i.e, each in 5 deg increments) in order (the up key for counterclockwise, and the down key for clockwise) until subjects found one that matched the remembered orientation. The central fixation was removed while subjects were going through the possible target orientations and re-appeared once they selected a target item by pressing the "enter" key. The next trial began 1 second following the subject's response. Subjects were seated approximately $60 \mathrm{~cm}$ from the computer screen. The same subjects completed 3 blocks of 48 trials each in both the simultaneous and sequential encoding conditions. These conditions were blocked and counterbalanced across subjects.

Experiment $\mathbf{1 b}$-The procedure was the same as Experiment 1a except for a few changes in the sample duration and subjects' response. The sample duration was reduced to $125 \mathrm{~ms}$ for the simultaneous condition. The sequential condition showed one stimulus at a time for $125 \mathrm{~ms}$ each with a 300ms inter-stimulus interval. Unlike Experiment 1a, subjects responded with a mouse click on the tested item to indicate the target orientation. The same subjects completed 7 blocks of 48 trials in each encoding condition. Encoding conditions were blocked and counterbalanced across observers.

\section{Results and discussion}

\section{Experiment 1a}

The key dependent variable was the offset between the subject's response and the orientation of the critical stimulus in the sample array. We observed, in the simultaneous encoding condition, a small but reliable reduction in mean offsets when items were presented across both hemifields $\left(\mathrm{M}=13.1^{\circ}, \mathrm{SD}=5.40\right)$ compared to those presented within the same hemifield $\left(\mathrm{M}=15.9^{\circ}, \mathrm{SD}=7.90\right), \mathrm{t}(13)=3.54 \mathrm{p}<.01$ (see Figure 3$)$. Thirteen of 14 subjects showed this bilateral advantage (see Figure 4 for the error distributions across all subjects). Critically, the sequential condition also revealed a significant bilateral advantage; recall errors for the bilateral condition were significantly smaller $\left(\mathrm{M}=13.1^{\circ}, \mathrm{SD}=5.56\right)$ than for the unilateral condition $\left(\mathrm{M}=15.6^{\circ}, \mathrm{SD}=6.43\right), \mathrm{t}(13)=2.41, \mathrm{p}=.03$ (see Figure 3). Eleven out of 14 subjects showed this effect (see Figure 5 for the error distributions across all subjects). An analysis of variance with the sample positions (uni- or bi-lateral) and the encoding conditions (simultaneous or sequential) as factors revealed a significant main effect of sample position with reduced mean offsets for the bilateral $\left(\mathrm{M}=13.1^{\circ}, \mathrm{SD}=1.43\right)$ compared to the unilateral $\left(\mathrm{M}=15.8^{\circ}, \mathrm{SD}=1.88\right)$ condition, $\mathrm{F}(1,13)=11.1, \mathrm{p}=.01$. There was no significant main effect of encoding, $\mathrm{F}(1,13)=$. $06, \mathrm{p}=.8$, or interaction between the two factors, $\mathrm{F}(1,13)=.22, \mathrm{p}=.65$. Thus, the results suggest a modest, but reliable bilateral advantage for the maintenance of orientation in visual WM. Furthermore, an equivalent bilateral advantage in the simultaneous and sequential conditions favors our argument that the effect was due to enhanced storage in memory per se rather than enhanced encoding efficiency.

\section{Experiment 1b}

Just as in Experiment 1a, a bilateral advantage was replicated for both encoding conditions (see Figure 6). The simultaneous condition resulted in a bilateral advantage of $4.3^{\circ}$, with smaller mean offsets for the bilateral condition $\left(\mathrm{M}=11.7^{\circ}, \mathrm{SD}=3.39\right)$ than the unilateral condition $\left(\mathrm{M}=16^{\circ}, \mathrm{SD}=7.01\right), \mathrm{t}(29)=4.44, \mathrm{p}<.01$. This effect was seen in 25 out of 30 subjects (see Figure 7 for the error distributions across all subjects). Likewise, the sequential condition 
yielded a reliable bilateral advantage of $2.1^{\circ}$, with smaller mean offsets for the bilateral $\left(\mathrm{M}=12.3^{\circ}, \mathrm{SD}=4.11\right)$ than the unilateral condition $\left(\mathrm{M}=14.4^{\circ}, \mathrm{SD}=5.10\right), \mathrm{t}(29)=5.34, \mathrm{p}<.01$. Twenty-five out of 30 subjects showed this bilateral advantage (see Figure 8 for the error distributions across all subjects). An analysis of variance with the sample position and encoding condition as factors again revealed a significant main effect of the sample position, $\mathrm{F}(1,29)$ $=32.6, \mathrm{p}<.01$, with reduced mean offsets for the bilateral $\left(\mathrm{M}=12^{\circ}, \mathrm{SD}=.63\right)$ compared to the unilateral condition $\left(\mathrm{M}=15.2^{\circ}, \mathrm{SD}=.98\right)$. There was no main effect of encoding. However, unlike Experiment 1a, a significant interaction between sample position and encoding was observed, $\mathrm{F}(1,29)=5.14, \mathrm{p}=.03$. A separate analysis revealed a smaller bilateral advantage in the sequential condition $\left(\mathrm{M}=2.10^{\circ}, \mathrm{SD}=2.16\right)$ than in the simultaneous condition $\left(\mathrm{M}=4.27^{\circ}\right.$, $\mathrm{SD}=5.26), \mathrm{t}(29)=2.27, \mathrm{p}=.03$. This suggests that part of the bilateral advantage observed the simultaneous condition may have been due to enhanced encoding rather than solely due to memory storage. Nevertheless, the reliable bilateral advantage in the sequential condition demonstrates a bilateral advantage even when the local display characteristics are identical between the bilateral and unilateral conditions.

These arguments notwithstanding, we also considered the possibility that even in the sequential condition, the encoding of the second item may have been affected by whether subjects were engaged in ongoing maintenance of items in the same or opposite hemifield. To test this possibility, we examined whether a reliable bilateral advantage was evident when only the data from the first items presented in the sequential condition were included. To reiterate our rationale, the first items presented in the sequential condition were identical for both the uniand bi-lateral displays, since they were presented one hemifield (quadrant) at a time. Thus, because there was no ongoing memory load when the first items were presented, encoding conditions for those items were precisely the same in the bilateral and unilateral conditions. Thus, a bilateral advantage for the first items presented would provide a conclusive disconfirmation of the hypothesis that encoding differences are the sole source of the advantage. To maximize statistical power, we combined the data from Experiments 1a and $1 \mathrm{~b}$. This analysis revealed a significant bilateral advantage for the item presented first in the sequential encoding conditions, $\mathrm{t}(43)=5.92, \mathrm{p}<.01$, showing reduced mean offsets for the bilateral $\left(\mathrm{M}=13.6^{\circ}, \mathrm{SD}=5.2\right)$ compared to the unilateral $\left(\mathrm{M}=16.9^{\circ}, \mathrm{SD}=7.65\right)$ condition. A similar bilateral advantage was marginally significant for the second item presented in the sequential encoding condition, $\mathrm{t}(43)=1.89, \mathrm{p}=.07$. An analysis of variance showed a significant main effect of order, such that mean offsets for the second sequence of sample array $(\mathrm{M}=12.1$, $\mathrm{SD}=.67)$ was smaller than those for the first sequence $(\mathrm{M}=15.3, \mathrm{SD}=.95), \mathrm{F}(1,43)=20.51, \mathrm{p}<$. 01 . There was a significant interaction between order and sample positions, such that the bilateral advantage was larger for the item presented first $(\mathrm{M}=3.3, \mathrm{SD}=3.71)$, than for the item presented second $(\mathrm{M}=1.0, \mathrm{SD}=3.72), \mathrm{F}(1,43)=8.92, \mathrm{p}=.01$. To conclude, the results from Experiments $1 \mathrm{a}$ and $1 \mathrm{~b}$ demonstrated enhanced performance in a working memory task when incoming visual information was distributed across the two visual hemifields as opposed to when the same information was presented unilaterally. This bilateral advantage was reliable when the sample items were presented sequentially, such that local display conditions were identical in the bilateral and unilateral conditions. Moreover, the bilateral advantage was reliable for the first items presented in the sequential condition when both local display conditions and ongoing storage in visual WM were equivalent across the bilateral and unilateral conditions. Thus, the observed bilateral advantage could not be explained by differences in encoding quality. These results corroborate previous findings of a bilateral advantage in a visual WM task (Delvenne, 2005) while also providing more conclusive evidence that the bilateral advantage can affect post-encoding stages such as storage in WM. 


\section{Experiments $2 \mathrm{a} \& \mathbf{2 b}$}

In the second set of experiments, we sought to further characterize how the bilateral advantage affects storage in WM. Specifically, recent research has suggested that capacity in visual working memory may be best understood by distinguishing between the maximum number of items that can be stored and the resolution or precision of each of those representations. For example, $\mathrm{Xu}$ and Chun (2006) found that distinct neural regions were sensitive to the number of items stored on the one hand (inferior intraparietal sulcus), and the complexity of the stored items on the other hand (superior parietal and lateral occipital cortex). Given that more complex items require more detailed representations to support accurate memory-guided behavior, these data suggest that different neural processes determine how many items can be stored and how much detail is maintained for the stored items. In line with these findings, Awh, Barton and Vogel (2007) examined individual differences in the number and resolution of the representations stored in WM and found no correlation between these two measures (though the reliability of each measure was confirmed). These data suggest a two-factor model in which number and resolution represent distinct aspects of memory ability. From this perspective, there are at least two distinct ways to explain enhanced storage during the bilateral condition in the present experiments. The bilateral advantage may have arisen because more items were stored from both hemifields. Alternatively, even if the same number of items was stored, those presented bilaterally might have been maintained with a higher precision. Hence in the final experiments, we attempted to determine which aspect of storage was influenced by the bilateral advantage.

To test whether the observed bilateral advantage was due to an increase in the number of items stored, or to the resolution with which these items were stored, we used an analytic approach developed by Zhang and Luck (2008) that provides independent estimates of the number and precision of the representations stored in visual WM. To detail their procedure, they employed a recall paradigm in which subjects remembered multiple colors for a brief period of time, and were asked to report the color of a probed item by clicking on a color wheel shown on a test array. They predicted that if visual WM memory is subject to discrete capacity limits - such that some information is retained regarding a limited number of items while zero information was retained for items exceeding the item limit - then response errors in this procedure should fall into two distinct categories. When the item was stored in WM, subjects' responses should be most frequent around the value of the probed item, with monotonic reductions in response frequency as the distance from the correct angle increases. By contrast, when the probed item was not stored, response errors should be randomly and evenly distributed across the full range of possible error values. Thus, the overall distribution of response errors should reveal a mixture of these two distributions. Their findings strongly corroborated these assumptions, showing that the distribution of response errors was best described by a mixture model that included two key parameters representing the probability that the probed item was in memory $(\mathrm{P}(\mathrm{mem})$, based on the probability of random responses), and the precision with which the probed item was held in memory (based on the standard deviation of response errors when subjects did have information about the critical stimulus.). We utilized this method in our final experiment to examine if the observed bilateral advantage reflected the number of items stored, or the precision of stored representations. We used the same stimuli as those in Experiment 1b, but increased the total number of items in the sample array to four to minimize ceiling effects in the estimates of how many items were stored. The stimuli were presented simultaneously in Experiment 2a, while they were presented sequentially (one pair at a time) in Experiment 2b. 


\section{Method}

Subjects

Two separate groups of subjects ( 25 for Experiment 2a, and 30 for Experiment $2 b$ ) participated in a one hour experimental session for a psychology course-credit at the University of Oregon. All subjects reported to have normal or corrected-to-normal vision.

\section{Stimuli}

The same stimuli as Experiment $1 \mathrm{~b}$ were used, and the number of stimuli was increased to four. Each stimulus was $4.6^{\circ}$ in diameter, and was presented on an imaginary half-circle, with two stimuli in each quadrant (see Figure 9). All stimuli appeared $7.4^{\circ}$ from the central fixation point. Adjacent stimuli were $45^{\circ}$ apart from each other and $22.5^{\circ}$ from either the horizontal or vertical meridian. Stimuli were presented on a gray background simultaneously in Experiment $2 \mathrm{a}$ or two at a time within the same quadrant in Experiment $2 \mathrm{~b}$.

\section{Procedure}

The same procedure as in Experiment $1 \mathrm{~b}$ was used for both Experiments $2 \mathrm{a}$ and $2 \mathrm{~b}$ with $\mathrm{a}$ reduction in the delay period from 1 second to $900 \mathrm{~ms}$. The difference between Experiment 2a and $2 \mathrm{~b}$ was the use of simultaneous (Experiment $2 \mathrm{a}$ ) versus sequential (Experiment $2 \mathrm{~b}$ ) presentations of the memoranda. All four stimuli were presented simultaneously for $500 \mathrm{~ms}$ in Experiment 2a, whereas in Experiment 2b, the stimuli were presented two at a time within a single quadrant for $300 \mathrm{~ms}$ each with a $600 \mathrm{~ms}$ inter-stimulus interval (like in Experiment 1, a trial duration was longer for the sequential than the simultaneous condition). After a $900 \mathrm{~ms}$ delay period, subjects responded with a mouse click on a circular probe to indicate the orientation of the item that was presented in the same position. The next trial began $1500 \mathrm{~ms}$ post response. Each experiment included 8 blocks of 48 trials.

\section{Results Exp.2a \& 2b}

Consistent with the previous results, both encoding conditions resulted in a significant bilateral advantage. A two (simultaneous versus sequential) by two (bilateral versus unilateral) analysis of variance revealed a main effect of sample position such that the mean offsets were significantly smaller for the bilateral condition $\left(\mathrm{M}=44.4^{\circ}, \mathrm{SD}=1.78\right)$ than for the unilateral condition $\left(\mathrm{M}=48.8^{\circ}, \mathrm{SD}=1.79\right), \mathrm{F}(1,53)=42.85, \mathrm{p}<.01$. There was a marginally significant effect of encoding condition, such that the mean offsets were larger for the simultaneous condition in Exp. $2 \mathrm{a}\left(\mathrm{M}=49.9^{\circ}, \mathrm{SD}=2.59\right)$ than for the sequential presentation in Exp. $2 \mathrm{~b}$ $\left(\mathrm{M}=43.3^{\circ}, \mathrm{SD}=2.36\right), \mathrm{F}(1,53)=3.59, \mathrm{p}=.06$. The interaction between the two factors was significant, $F(1,53)=4.08, p=.05$, indicating an amplified bilateral advantage in the simultaneous condition. Nevertheless, a simple t-test revealed a significant bilateral advantage in both the simultaneous $\left(\mathrm{M}=47^{\circ}, \mathrm{SD}=12.47\right.$ and $\mathrm{M}=52.8^{\circ}, \mathrm{SD}=11.60$, for the bilateral and unilateral condition, respectively), $\mathrm{t}(24)=5.2, \mathrm{p}<.01$, and sequential conditions $\left(\mathrm{M}=41.8^{\circ}\right.$, $\mathrm{SD}=13.64$ and $\mathrm{M}=44.8^{\circ}, \mathrm{SD}=14.4$, for the bilateral and unilateral condition, respectively), $\mathrm{t}$ (29) $=3.75, p<.01$ (see Figure 10). These results again confirm that the bilateral advantage is not driven by differences in encoding alone.

The key result came from using the analytic procedure developed by Zhang and Luck (2008) to independently measure the number and precision of the representations stored in the bilateral and unilateral conditions. This analysis revealed that the bilateral advantage was due to a reliable difference in the number $\left(\mathrm{P}_{\mathrm{mem}}\right)$, but not the resolution (SD) of the stored representations. This result was obtained in both the simultaneous and sequential conditions (see Figure 11 for $\mathrm{P}_{\mathrm{mem}}$ and Figure 12 for $\mathrm{SD}$ ). A simple $\mathrm{t}$-test for the simultaneous encoding condition in Exp.2a showed a significantly higher probability that the probed items were in 
memory (i.e., $\left.\mathrm{P}_{\mathrm{mem}}\right)$ for the bilateral display $(\mathrm{M}=.57, \mathrm{SD}=.17)$ than for the unilateral display $(\mathrm{M}=.51, \mathrm{SD}=.15), \mathrm{t}(24)=-3.67, \mathrm{p}<.01$, but not for the precision of the probed items (i.e., $\mathrm{SD}$ ), $\mathrm{p}=.65$. The sequential encoding condition in Exp. $2 \mathrm{~b}$ revealed the same pattern such that $\mathrm{P}_{\text {mem }}$ was significantly higher for the bilateral display $(\mathrm{M}=.65, \mathrm{SD}=.16)$ than for the unilateral display $(\mathrm{M}=.61, \mathrm{SD}=.18), \mathrm{t}(29)=-2.51, \mathrm{p}=.02$. Again, mnemonic precision $(\mathrm{SD})$ in the sequential condition was equivalent between the bilateral and unilateral displays, $\mathrm{p}=.46$. A 2 (encoding condition) by 2 (sample display) analysis of variance on $\mathrm{P}_{\text {mem }}$ revealed a significant main effect of sample display, with higher $\mathrm{P}_{\mathrm{mem}}$ for the bilateral display $(\mathrm{M}=.61, \mathrm{SD}=.02)$ than the unilateral display $(\mathrm{M}=.56, \mathrm{SD}=.02), \mathrm{F}(1,53)=19.82, \mathrm{p}<.01$. There was also a significant main effect of encoding condition, with higher $\mathrm{P}_{\mathrm{mem}}$ for the sequential condition $(\mathrm{M}=.63, \mathrm{SD}=$. $03)$ than for the simultaneous condition $(\mathrm{M}=.54, \mathrm{SD}=.03), \mathrm{F}(1,53)=4.60, \mathrm{p}=.04$. The interaction between the two factors was not significant, indicating that the size of a bilateral advantage did not depend on whether the items were presented simultaneously or sequentially.

Further analysis showed that our results were well explained by the mixture model of Zhang and Luck (2008), containing the two components, P(mem) and S.D. A uniform Gaussian distribution alone, whose width should correspond to the precision of stored item, could not account for the observed results as well as the mixture model for both Experiment $2 \mathrm{a}$ and $2 \mathrm{~b}$ (see Figure 13 and 14, respectively). More specifically, although a pure Gaussian distribution explained a moderate amount of variance for both the uni- and bi-lateral conditions in Experiment $2 \mathrm{a}\left(\mathrm{R}^{2}=.65\right.$ and $\mathrm{R}^{2}=.68$, respectively), it differed significantly from the observed distribution (at $.01 \alpha$ level on Kolmogorov-Smirnov (k-s) test). The mixture model resulted in a better fit for both display types $\left(\mathrm{R}^{2 \mathrm{R}}=.99\right.$ and $\mathrm{p}>.1 \mathrm{on} \mathrm{k}-\mathrm{s}$ test for both display types). Likewise in Experiment $2 b$, the same amount of variance was explained by the mixture model $\left(\mathrm{R}^{2}=.99\right.$ and p>.1 on k-s test for both display types), and less variance was explained by a pure Gaussian $\left(\left(\mathrm{R}^{2}=.72\right.\right.$ and $\mathrm{R}^{2}=.74$ for the uni- and bi-lateral displays, respectively), with poorer fit ( .01 alpha level on k-s test for both display types).

Although these findings conform well to the assumptions of the mixture model, we also considered the alternative view (Bays, Catalao, and Hussain, 2009) that the putative random component of the response distribution postulated in the mixture model could be largely explained by cases in which subjects accidentally reported the value of a different item from the one probed (i.e., mislocalization errors). Given that the values between stimuli varied randomly, such errors would produce seemingly random responses, even if subjects had stored the probed item. Bays et al. tested this hypothesis by examining whether there were reliable tendencies for observers to report non-target values during a recall procedure modeled after that used by Zhang and Luck (2008). Indeed, their findings revealed that a preponderance of the responses that were randomly related to the target value could be accounted for by a tendency to report values from other stimuli in the sample array. Hence, we re-analyzed Experiment 2 with the goal of determining the proportion of response errors that could be explained by a tendency to report the value of non-target items from within the same sample array. In this analysis we calculated the deviation between each individual trial response and each of the three non-target values in that trial. According to the Bays et al. (2009) hypothesis, this should reveal a clear association between subject responses and non-target values, such that the preponderance of individual subject responses could be attributed to either target or non-target values from the corresponding trial. Our findings were not fully in line with these predictions. One issue we encountered was the number of subjects whose response distributions for the non-probed items were so random (i.e., no evidence that responses were centered around distractor values) that the model could not compute the appropriate parameter values (13 out of 25 subjects in Experiment 2a, and 9 out of 30 subjects in Experiment 2b). By contrast, every subject tested could be effectively described by the mixture model when only the target values were used to compute response offsets. Therefore, our analysis here was based on the aggregate data across all subjects, rather than on individual basis. Overall, we found a non-zero but modest 
tendency for subjects to report the values of the non-probed items. Critically for the present purposes, the probability of reporting non-target values was equivalent in the unilateral and bilateral conditions. In the simultaneous condition of Experiment 2a, only $2 \%$ of subjects' responses could be attributed to the accidental report of non-target values in both the unilateral and bilateral conditions. In the sequential condition of Experiment $2 b$, the rates somewhat increased, and $6 \%$ and $5 \%$ of response offsets could be attributed to non-target reports in the unilateral and bilateral conditions, respectively ${ }^{1}$. In the sequential condition, however, since the $1 \%$ higher misreport was in the unilateral condition, we examined its significance further to see if the higher incidence of reporting one of the non-probed items in the unilateral condition had indeed driven the observed bilateral advantage. For this analysis, we used individual data and re-examined a bilateral advantage without these subjects who showed a higher probability of reporting non-target values in the unilateral condition. If such misreport was the source of the bilateral advantage, excluding their data should eliminate the observed bilateral advantage. Inconsistent with this hypothesis, the bilateral advantage remained reliable when all subjects who showed an increased tendency to report non-target values in the unilateral condition were removed from the analysis. Thus, the bilateral advantage for memory could not be explained by differences in the frequency of reporting non-target values.

Finally, the results of Experiments $2 \mathrm{~b}$ replicated the key finding in Experiments $1 \mathrm{a}$ and $1 \mathrm{~b}$ that there was a significant bilateral advantage even when the analysis was restricted to only the items presented first in the sequential condition. There was a reliable bilateral advantage for the items presented first in the sequential condition, $\mathrm{t}(29)=2.95, \mathrm{p}=.01,\left(\mathrm{M}=52.1^{\circ}, \mathrm{SD}=15.4\right.$ and $\mathrm{M}=55.8^{\circ}, \mathrm{SD}=15.9$ in the mean offsets for the bilateral and unilateral condition, respectively). Likewise, a significant bilateral advantage was also found for the items presented second in the sequential condition, $\mathrm{t}(29)=2.77, \mathrm{p}=.01,\left(\mathrm{M}=31.3^{\circ}, \mathrm{SD}=14.8\right.$ and $\mathrm{M}=33.9^{\circ}$, $\mathrm{SD}=16.6$ in the mean offsets for the bilateral and unilateral condition, respectively). The interaction between order and sample position was not significant, indicating that the size of the bilateral advantage was equivalent for the items presented first and second. There was, however, a significant main effect of order such that the mean offsets were smaller for the items presented second $(\mathrm{M}=32.6, \mathrm{SD}=2.83)$ than for the items presented first $(\mathrm{M}=54.0, \mathrm{SD}=2.79), \mathrm{F}$ $(1,29)=77.12, \mathrm{p}<.01$. These results underscore the previous conclusions by demonstrating that encoding differences cannot explain the bilateral advantage in this task.

\section{General Discussion}

Past studies have shown enhanced performance when visual information is presented in both the right and left visual hemifields, as opposed to when the same amount of information is presented in one hemifield. This bilateral advantage has been demonstrated in several tasks requiring a rapid initial encoding (e.g., Awh and Pashler, 2000; Reuter-Lorenz, Stanczak and Miller, 1999; Sereno and Kosslyn, 1991). The most striking demonstration, however, came from a multiple object tracking procedure (Alvarez and Cavanagh, 2005) that found a doubling of tracking capacity in the bilateral condition. Although various studies have suggested overlap in the key cognitive processes that mediate tracking and WM storage, a bilateral advantage in working memory has not been consistently demonstrated. The present study provides conclusive evidence for a bilateral advantage in visual working memory for orientations while ruling out the possibility that differences in encoding were responsible for superior performance in the bilateral condition. This encoding explanation was refuted through the demonstration of a bilateral advantage even when the stimuli were presented one hemifield

\footnotetext{
${ }^{1} \mathrm{We}$ also analyzed the sequential condition of Experiment $2 \mathrm{~b}$ in terms of non-probed items separately for those presented together with the target item versus those that appeared on a different sample display from the target's. Again, our results stayed unchanged, such that incidence of mislocalization was indistinguishable between the uni- and bi-lateral presentations regardless of whether the items were presented with targets or not.
} 
(i.e., quadrant) at a time in both the bilateral and unilateral conditions. Moreover, the bilateral advantage in this sequential condition was still evident when only the items presented first were included in the analysis. This analysis provides a stronger test of whether the bilateral advantage would be robust in the absence of encoding differences, given that encoding of the items presented second could theoretically have been affected by the concurrent maintenance of items that had been presented in the same visual field. Thus, the maintenance of stimulus orientations in visual WM is enhanced by bilateral presentations of the memoranda.

We further inquired into which aspect of memory storage was facilitated by the bilateral presentation of stimuli. The bilateral advantage could be due to the storage of a larger number of items when the sample display was distributed across both the left and right hemifields. Alternatively, the advantage could reflect higher mnemonic precision in the bilateral condition. By utilizing an analytical procedure to separately measure the number and resolution of the stored representations (Zhang and Luck, 2008), we found that bilateral presentations led to a higher probability that the critical item was stored rather than a higher resolution of the stored representations. This finding may seem counterintuitive at first, given a presence of a bilateral advantage when the capacity estimates were presumably at near ceiling in Experiment 1 . Our working hypothesis is that even with sub-span arrays, there is a small probability that the critical item is dropped from memory by the time that subjects recall the probed value; thus, the bilateral advantage in such displays may reflect a reliable increase in such drops in the unilateral condition. Furthermore, our findings cannot be explained by an increased tendency to report non-target values in the unilateral condition (Bays et al., 2009). Although past studies have reported evidence of such non-target reports, the present data revealed very low rates of nontarget reports that were no more likely in the unilateral than in the bilateral condition. Thus, although there is already robust evidence for a bilateral advantage during the initial stages of target selection (Alvarez and Cavanagh, 2005), the present data suggest that later stages of processing such as maintenance and storage in WM are also enhanced by bilateral presentations of visual information.

Although we showed evidence of a bilateral advantage for memory storage, the effect was substantially smaller than the one observed by Alvarez and Cavanagh, who found a doubling of tracking capacity in the bilateral relative to the unilateral condition. Obviously, the modest effect sizes in the current work fall well short of that mark. Although our current study does not offer a clear explanation for this discrepancy, we can provide a few speculations. As Alvarez and Cavanagh (2005) and Delvenne (2005) suggested, the bilateral advantage may have influenced a process of stimulus selection that relies strongly on independent hemispheric resources. Indeed, past studies linking top-down selection processes and maintenance in visual WM (e.g., Awh and Jonides, 2001; Awh, Reuter-Lorenz, and Jonides, 1998, Awh, Vogel, and Oh, 2006) suggest that a similar selection through attention-based rehearsal process may also contribute to the subsequent storage of information in visual WM. One possibility is that brief interruptions in this ongoing selection process may have reliable but relatively small effects on the probability of successful maintenance, if the decay of unrehearsed information is relatively gradual. By contrast, even a momentary failure of selection while tracking moving objects could lead to immediate confusions with nearby distractor objects. This speculation raises a related possibility. Perhaps the bilateral advantage is amplified when there is strong competition from irrelevant distracters. The multiple object tracking procedures employed by Alvarez and Cavanagh (2005) required constant selection of incoming visual information due to sustained interference from irrelevant visual stimuli. By contrast, all items in the current memory tasks were relevant targets. Hence the presence of to-be-suppressed information in the tracking task might have increased the magnitude of a bilateral advantage. Future research can examine whether the magnitude of the bilateral advantage is modulated by interference during encoding or maintenance phases of working memory tasks. 
It is also important to consider why past studies have not yielded consistent evidence for a bilateral advantage in working memory or other attention tasks such as visual search. Prior to the present findings, the study by Delvenne (2005) has provided the only clear evidence of a bilateral advantage in a visual working memory task. However, there are two important aspects of the literature that are worth noting. First, past failures to obtain a bilateral advantage in working memory had examined memory for color (Delvenne, 2005; Xu and Nakayama, 2007). In line with these null results, unpublished data from our own laboratory found no trace of a bilateral advantage in color memory procedures, even when the same recall procedure as our current task was employed. It is possible that a bilateral advantage only influences storage success within specific stimulus domains. Delvenne's procedures required memory for absolute stimulus position, while ours required memory for orientation; from the perspective of the dorsal/ventral dichotomy in visual perception (Ungerleider and Mishkin, 1982) perhaps the bilateral advantage affects memory for dorsal rather than ventral stream visual properties. This speculation falls in line with the suggestion from Alvarez and Cavanagh (2005) that the bilateral advantage may primarily influence how attention is deployed to spatial information, as is in the case of tracking and spatial memory task, but not to identity which visual search and color information necessitate. Clearly, further research is needed to determine the boundary conditions of this effect. A second factor that we suspect may be important is the specific experimental procedure that is used to detect the effect. Our own pilot work suggests that the change detection paradigm - despite its obvious utility for the study of many other aspects of WM - may not be a sensitive method to document a bilateral advantage. Although we have never failed to replicate the bilateral advantage for orientation using recall procedures such as those reported here, the same stimuli when used in a change detection procedure have not yielded consistent outcomes. Provided that a bilateral advantage for memory storage is markedly small, the change detection paradigm might obscure small effects due to its reliance on coarse two-alternative forced-choice responses.

To conclude, our results provide evidence that even a relatively late-stage cognitive process such as maintenance and storage in visual WM shows a benefit when incoming visual information is initially received by both hemispheres rather than by a single hemisphere. Although the current data do not offer direct evidence for complete hemifield independence in visual working memory, we showed a reliable bilateral advantage for the probability of storage, but not for the resolution of the stored representations. Future research will be necessary to characterize how the two hemispheres coordinate with one another to maximize the information that can be maintained in this online workspace.

\section{References}

Alvarez GA, Cavanagh P. Independent resources for attentional tracking in the left and right visual hemifield. Psychological Science 2005;16:637-643. [PubMed: 16102067]

Awh E, Barton B, Vogel E. Visual Working Memory Represents a Fixed Number of Items Regardless of Complexity. Psychological Science 2007;18:622-628. [PubMed: 17614871]

Awh E, Jonides J. Overlapping mechanisms of attention and spatial working memory. Trends in Cognitive Sciences 2001;5:119-126. [PubMed: 11239812]

Awh E, Jonides J, Reuter-Lorenz PA. Rehearsal in spatial working memory. Journal of Experimental Psychology: Human Perception and Performance 1998;24:780-790. [PubMed: 9627416]

Awh E, Pashler H. Evidence for split attentional foci. Journal of ExperimentalPsychology: Human Perception and Performance 2000;26:834-846.

Awh E, Vogel E, Oh S-H. Interactions between attention and working memory. Neuroscience 2006;139:201-208. [PubMed: 16324792]

Barton B, Ester E, Awh E. Discrete resource allocation in visual working memory. Journal of Experimental Psychology: Human Perception and Performance 2009;35:1359-1367. [PubMed: 19803642] 
Bays PM, Catalao RF, Husain M. The precision of visual working memory is set by allocation of a shared resource. Journal of Vision 2009;9:1-11. [PubMed: 19810788]

Carlson TA, Alvarez GA, Cavanagh P. Quadrantic deficit reveals anatomical constraints on selection. Proceedings of the National Academy of Sciences of the United States of America 2007;104:1349613500. [PubMed: 17673552]

Cavanagh P, Alvarez GA. Tracking multiple targets with multifocal attention. Trends in Cognitive Science 2005;9:349-354.

Cousineau D. Confidence intervals in within-subject designs: A simpler solution to Loftus and Masson's method. Tutorial in Quantitative Methods for Psychology 2005;1:42-45.

Culham JC, Brandt SA, Cavanagh P, Kanwisher NG, Dale AM, Tootell RBH. Cortical fMRI activation produced by attentive tracking of moving targets. Journal of Neurophysiology 1998;80:2657-2670. [PubMed: 9819271]

Culham JC, Cavanagh P, Kanwisher NG. Attention response functions: Characterizing brain areas using fMRI activation during parametric variations of attentional load. Neuron 2001;32:737-745. [PubMed: 11719212]

Delvenne J. The capacity of visual short-term memory within and between hemifields. Cognition 2005;96:B79-B88. [PubMed: 15996557]

Drew T, Vogel EK. Neural measures of individual differences in selecting and tracking multiple moving objects. Journal of Neuroscience 2008;28:4183-4191. [PubMed: 18417697]

Duncan J, Bundesen C, Olson A, Humphreys G, Chavda S, Shibuya H. Systematic analysis of deficits in visual attention. Journal of Experimental Psychology: General 1999;128:450-478. [PubMed: 10650583]

Fougnie D, Marois R. Distinct capacity limits for attention and working memory. Psychological Science 2006;17:526-534. [PubMed: 16771804]

Howe PD, Horowitz TS, Morocz IA, Wolfe J, Livingstone MS. Using fMRI to distinguish components of the multiple object tracking task. Journal of Vision 2009;9:1-11. [PubMed: 19757919]

Jovicich J, Peters RJ, Koch C, Braun J, Chang L, Ernst T. Brain areas specific for attentional load in a motion-tracking task. Journal of Cognitive Neuroscience 2001;13:1048-1058. [PubMed: 11784443]

Kraft A, Muller NG, Hagendorf H, Schira MM, Dick S, Fendrich RM, Brandt SA. Interactions between task difficulty and hemispheric distribution of attended locations: implications for the splitting attention debate. Cognitive Brain Research 2005;24:19-32. [PubMed: 15922154]

Kraft A, Pape N, Hagendorf H, Schmidt S, Naito A, Brandt SA. What determines sustained visual attention? The impact of distracter positions, task difficulty and visual fields compared. Brain Research 2007;1133:123-135. [PubMed: 17174284]

Linden DEJ, Bittner RA, Muckli L, Waltz JA, Kriegeskorte N, Goebel R, Singer W, Munk MHJ. Cortical capacity constraints for visual working memory: dissociation of fMRI load effects in a fronto-parietal network. NeuroImage 2003;20:1518-1530. [PubMed: 14642464]

Liu T, Jiang Y, Sun X, He S. Reduction of the crowding effect in spatially adjacent but cortically remote visual stimuli. Current Biology 2009;19:127-132. [PubMed: 19135367]

Luck SJ, Hillyard SA, Mangun GR, Gazzaniga MS. Independent hemispheric attentional systems mediate visual search in split-brain patients. Nature 1989;342:543-545. [PubMed: 2586625]

Luck SJ, Vogel EK. The capacity of visual working memory for features and conjunctions. Nature 1997;390:279-281. [PubMed: 9384378]

Muller MM, Malinowski P, Gruber T, Hillyard SA. Sustained division of the attentional spotlight. Nature 2003;424:309-312. [PubMed: 12867981]

Oksama L, Hyönä J. Is multiple object tracking carried out automatically by an early vision mechanism independent of higher-order cognition? An individual difference approach. Visual Cognition 2004;11:631-671.

Pashler H. Familiarity and visual change detection. Perception and Psychophysics 1988;44:369-378. [PubMed: 3226885]

Pylyshyn ZW, Storm RW. Tracking multiple independent targets: evidence for a parallel tracking mechanism. Spatial Vision 1988;3:179-197. [PubMed: 3153671] 
Reuter-Lorenz PA, Stanczak L, Miller AC. Neural recruitment and cognitive aging: Two hemispheres are better than one, especially as you age. Psychological Science 1999;10:494-500.

Scalf PE, Banich MT, Kramer AF, Narechania K, Simon CD. Double take: Parallel processing by the cerebral hemispheres reduces the attentional blink. Journal of Experimental Psychology: Human Perception and Performance 2007;33:298-329. [PubMed: 17469970]

Scolari M, Vogel E, Awh E. Perceptual Expertise Enhances the Resolution But Not the Number of Representations in Working Memory. Psychological Bulletin and Review 2008;15:215-222.

Sereno AB, Kosslyn SM. Discrimination within and between hemifields: A new constraint on theories of attention. Neuropsychologia 1991;29:659-675. [PubMed: 1944868]

Sperling G. The information available in brief visual presentations. Psychological Monographs: General and Applied 1960;74(11) Whole No. 498.

Todd JJ, Marois R. Capacity limit of visual short-term memory in human posterior parietal cortex. Nature 2004;428:751-754. [PubMed: 15085133]

Todd JJ, Marois R. Posterior parietal cortex activity predicts individual differences in visual short-term memory capacity. Cognitive, Affective, and Behavioral Neuroscience 2005;5:144-155.

Ungerleider, LG.; Mishkin, M. Two cortical visual systems.. In: Ingle, DJ.; Goodale, MA.; Mansfield, RJW., editors. Analysis of visual behavior. MIT Press; Cambridge, MA: 1982. p. 549-586.

Vogel EK, Machizawa MG. Neural activity predicts individual differences in visual working memory capacity. Nature 2004;428:749-751.

Vogel EK, McCollough AW, Machizawa MG. Neural measures reveal individual differences in controlling access to visual working memory. Nature 2005;438:500-503. [PubMed: 16306992]

Vogel EK, Woodman GF, Luck SJ. Storage of features, conjunctions, and objects in visual working memory. Journal of Experimental Psychology: Human Perception and Performance 2001;27:92-114. [PubMed: 11248943]

Xu Y, Chun MM. Dissociable neural mechanisms supporting visual short-term memory for objects. Nature 2006;440:91-95. [PubMed: 16382240]

Xu Y, Nakayama K. Visual short-term memory benefit for objects on different 3-D surfaces. Journal of Experimental Psychology: General 2007;136:653-662. [PubMed: 17999577]

Zhang W, Luck SJ. Discrete fixed-resolution representations in visual working memory. Nature 2008;453:233-235. [PubMed: 18385672] 


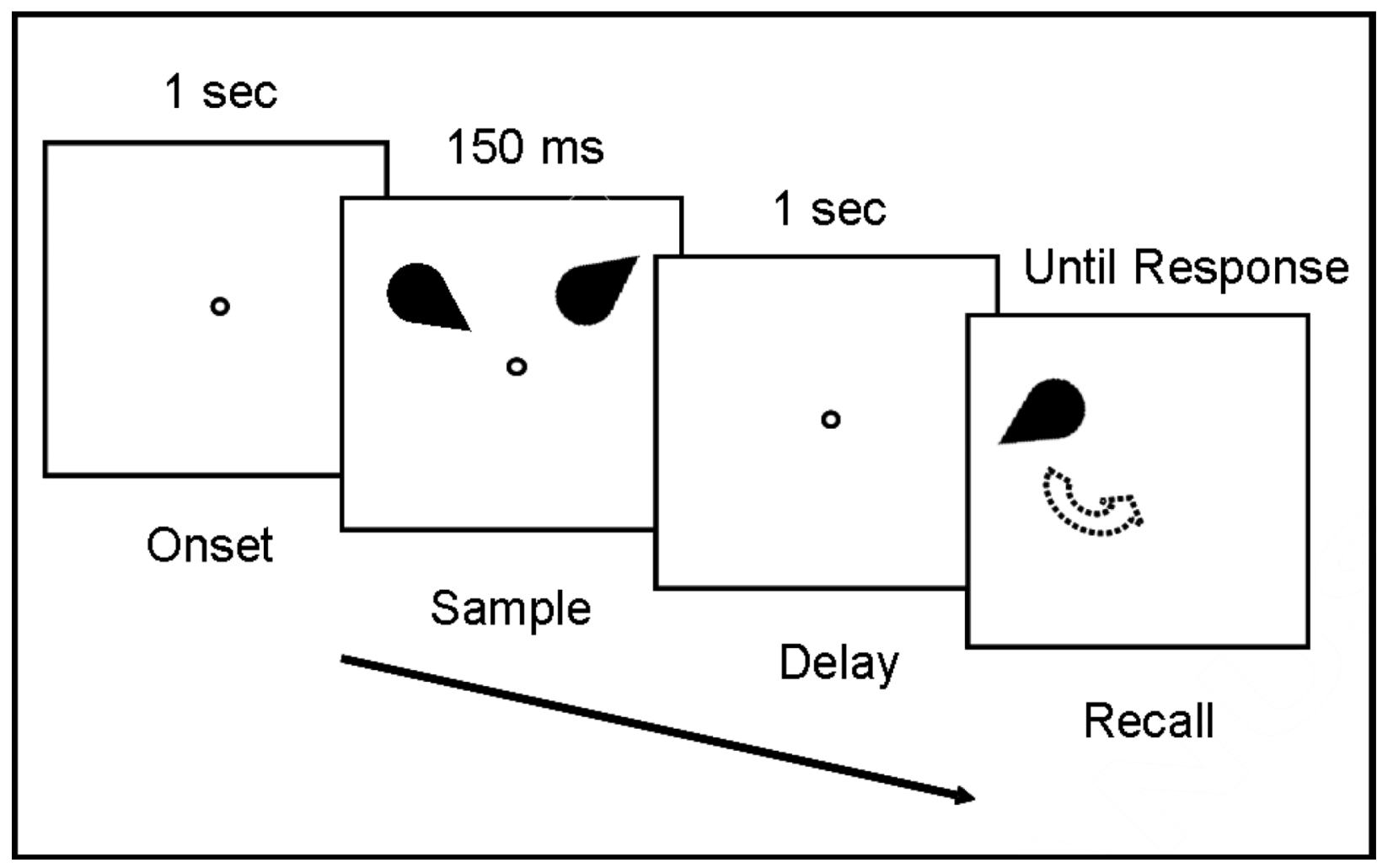

Figure 1.

The Sequence of events in a single trial of the recall procedure in Experiment 1a for the simultaneous condition. The dotted arrow indicates toward the correct position of the probed item. 


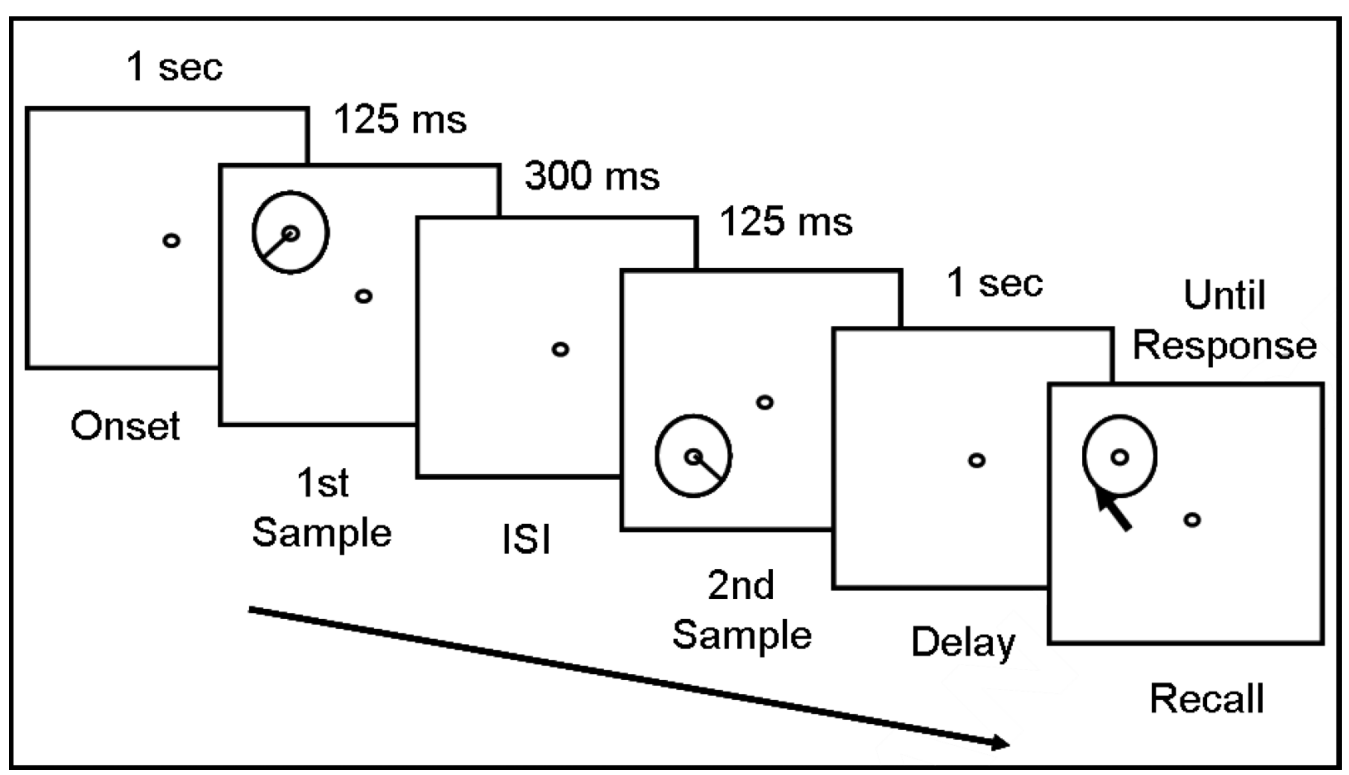

Figure 2.

The sequence of events in a single trial of the recall procedure in Experiment $1 \mathrm{~b}$ for the sequential condition. The arrow indicates the correct position of the probed item. 


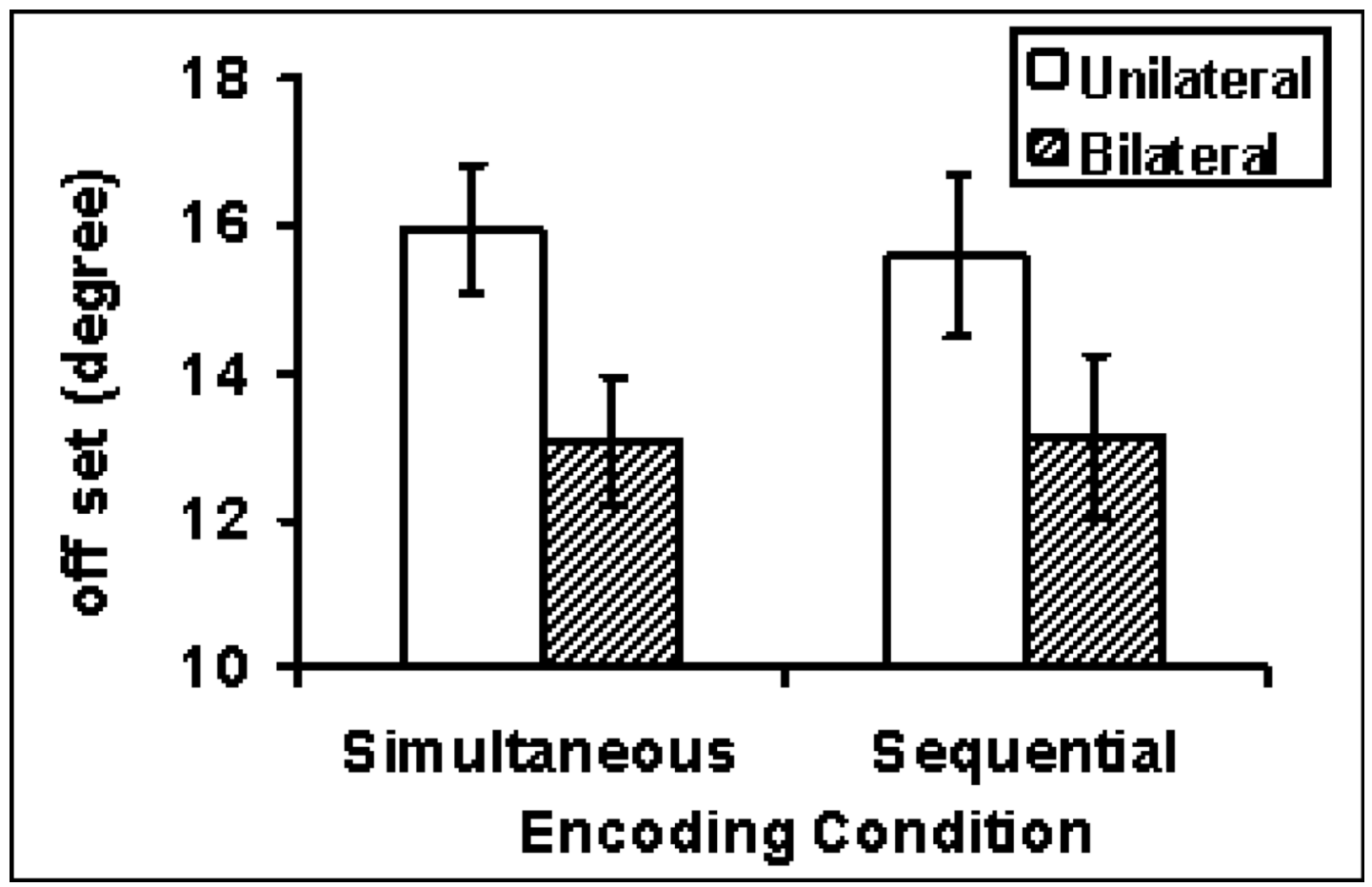

Figure 3.

Recall error for the unilateral and bilateral displays under the simultaneous and sequential encoding processes in Experiment 1a. The error bar represents 95\% confidence interval (Cousineau, 2005). 



Figure 4.

A distribution across all subjects for recall error for the unilateral and bilateral displays under the simultaneous encoding processes in Experiment 1a. 

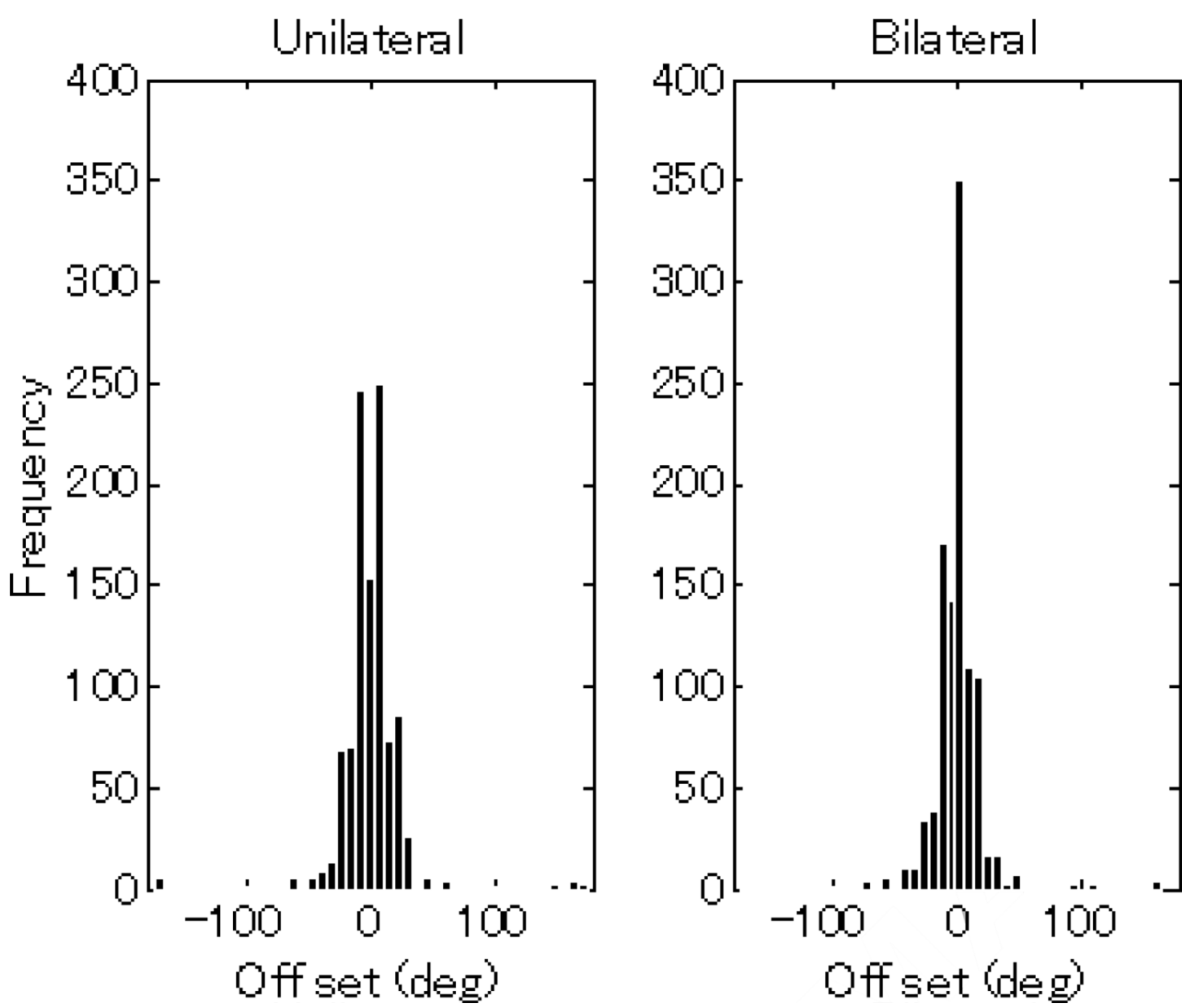

Figure 5.

A distribution across all subjects for recall error for the unilateral and bilateral displays under the sequential encoding processes in Experiment 1a. 


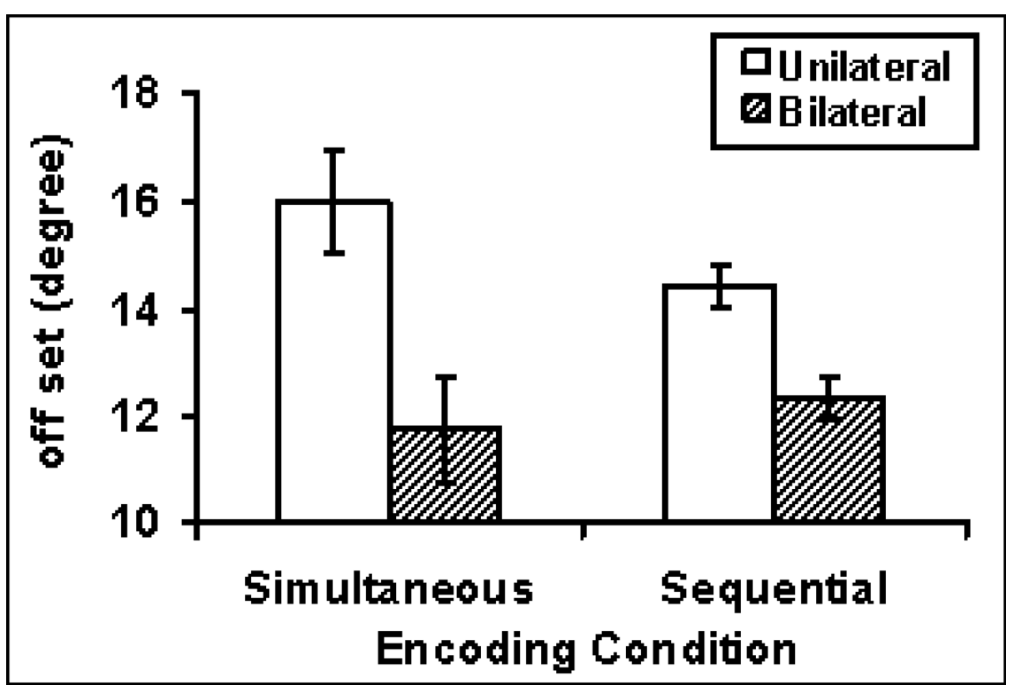

Figure 6.

Recall error for the unilateral and bilateral displays under the simultaneous and sequential encoding processes in Experiment 1b. The error bar represents 95\% confidence interval (Cousineau, 2005). 

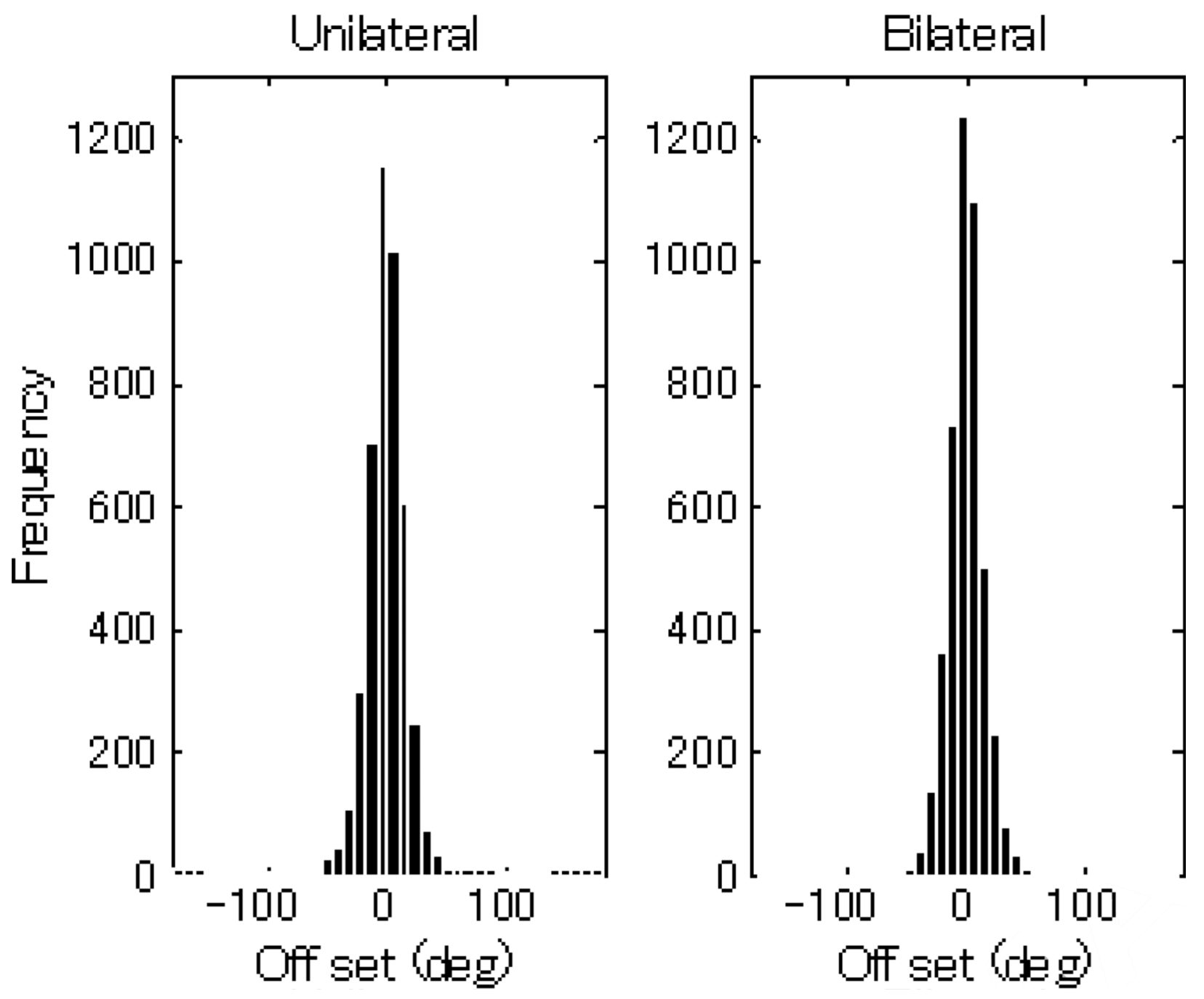

Figure 7.

A distribution across all subjects for recall error for the unilateral and bilateral displays under the simultaneous encoding processess in Experiment $1 \mathrm{~b}$. 

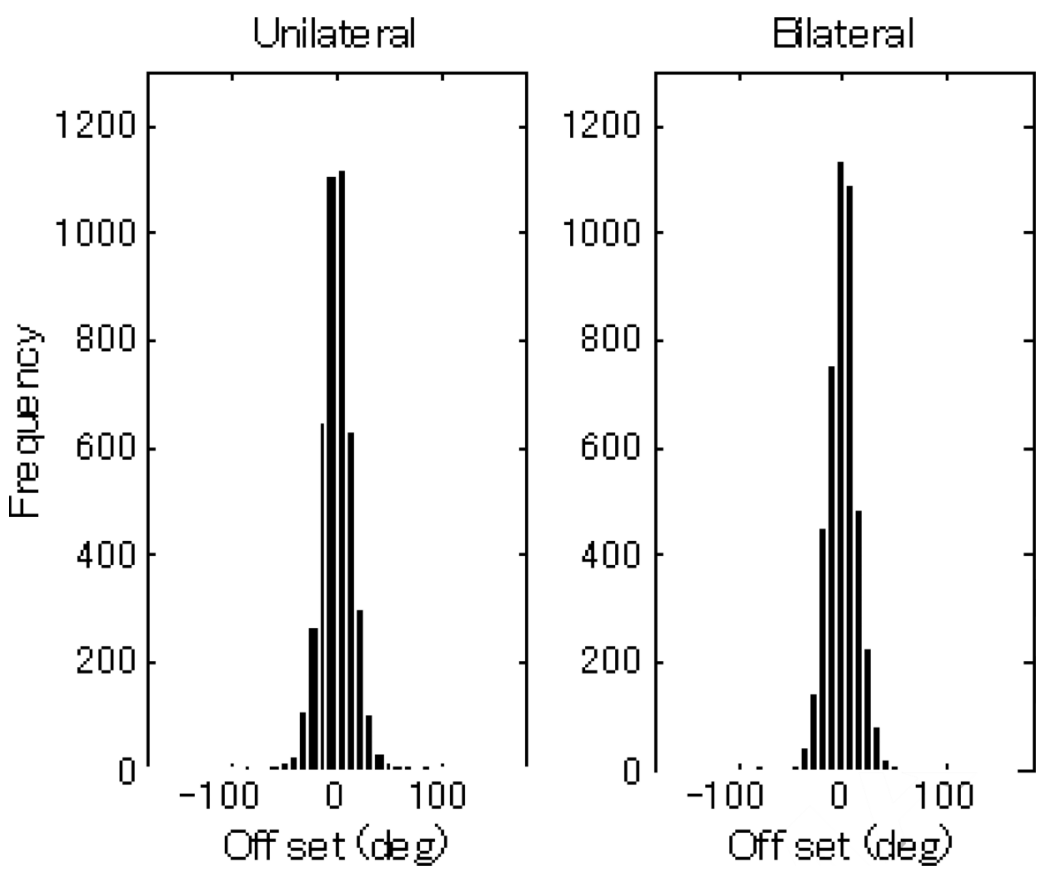

Figure 8.

A distribution across all subjects for recall error for the unilateral and bilateral displays under the sequential encoding processes in Experiment $1 \mathrm{~b}$. 


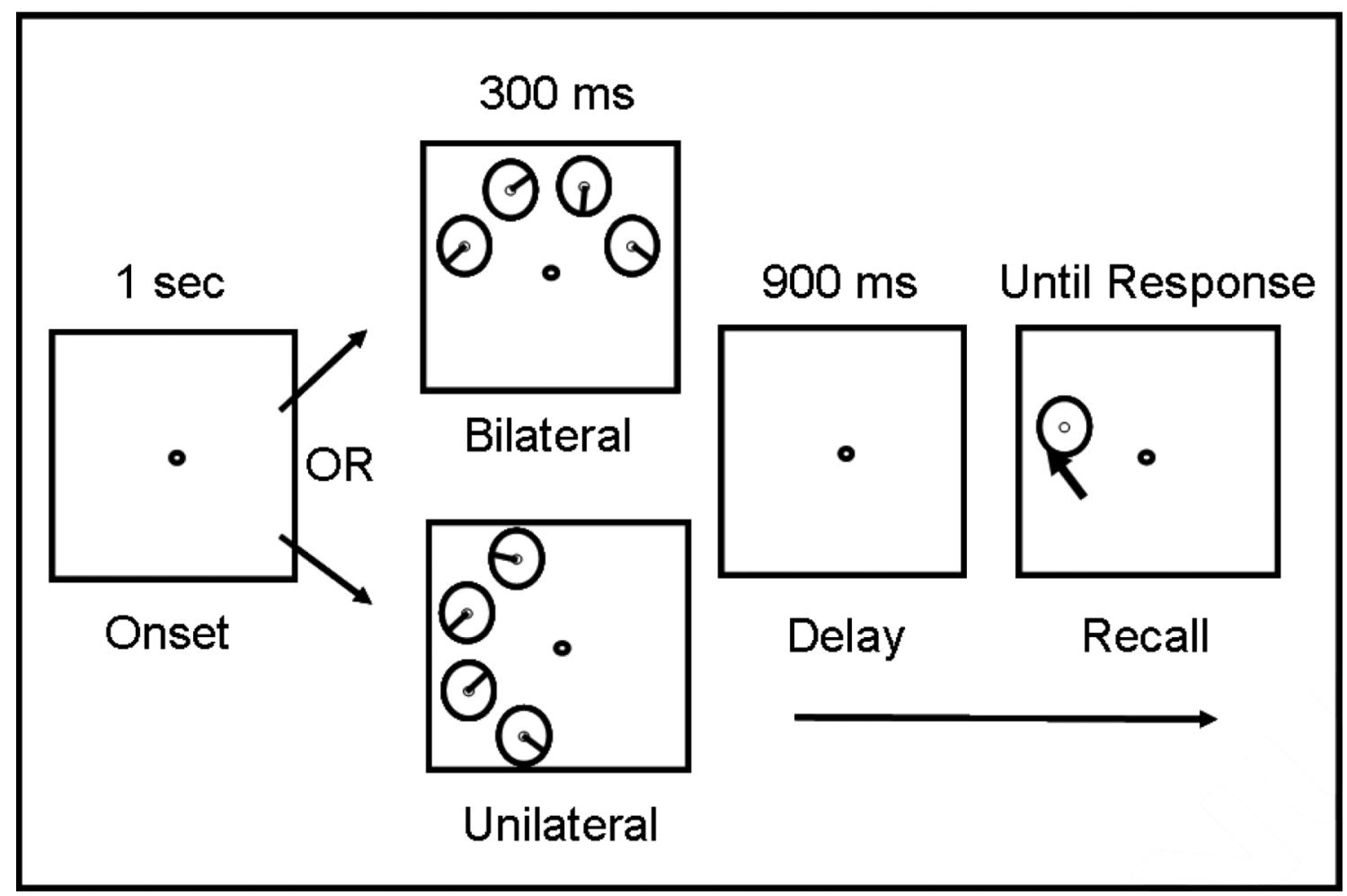

Figure 9.

The sequence of events in a single trial of the recall procedure for the simultaneous condition in Experiment 2a. 


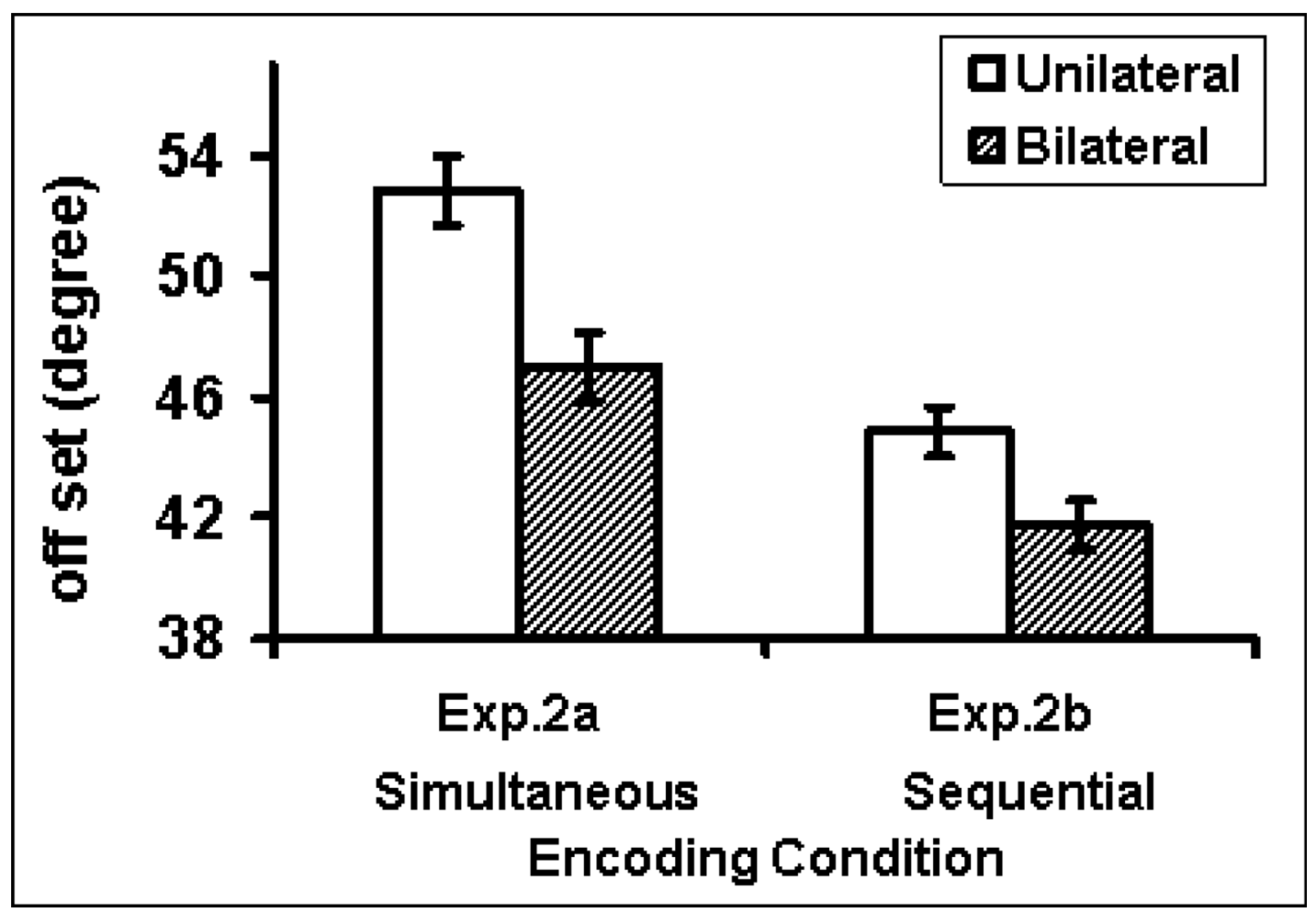

Figure 10.

Recall error for the unilateral and bilateral displays under the simultaneous and sequential encoding processes in Experiment $2 \mathrm{a}$ and $2 \mathrm{~b}$, respectively. The error bar represents $95 \%$ confidence interval (Cousineau, 2005). 


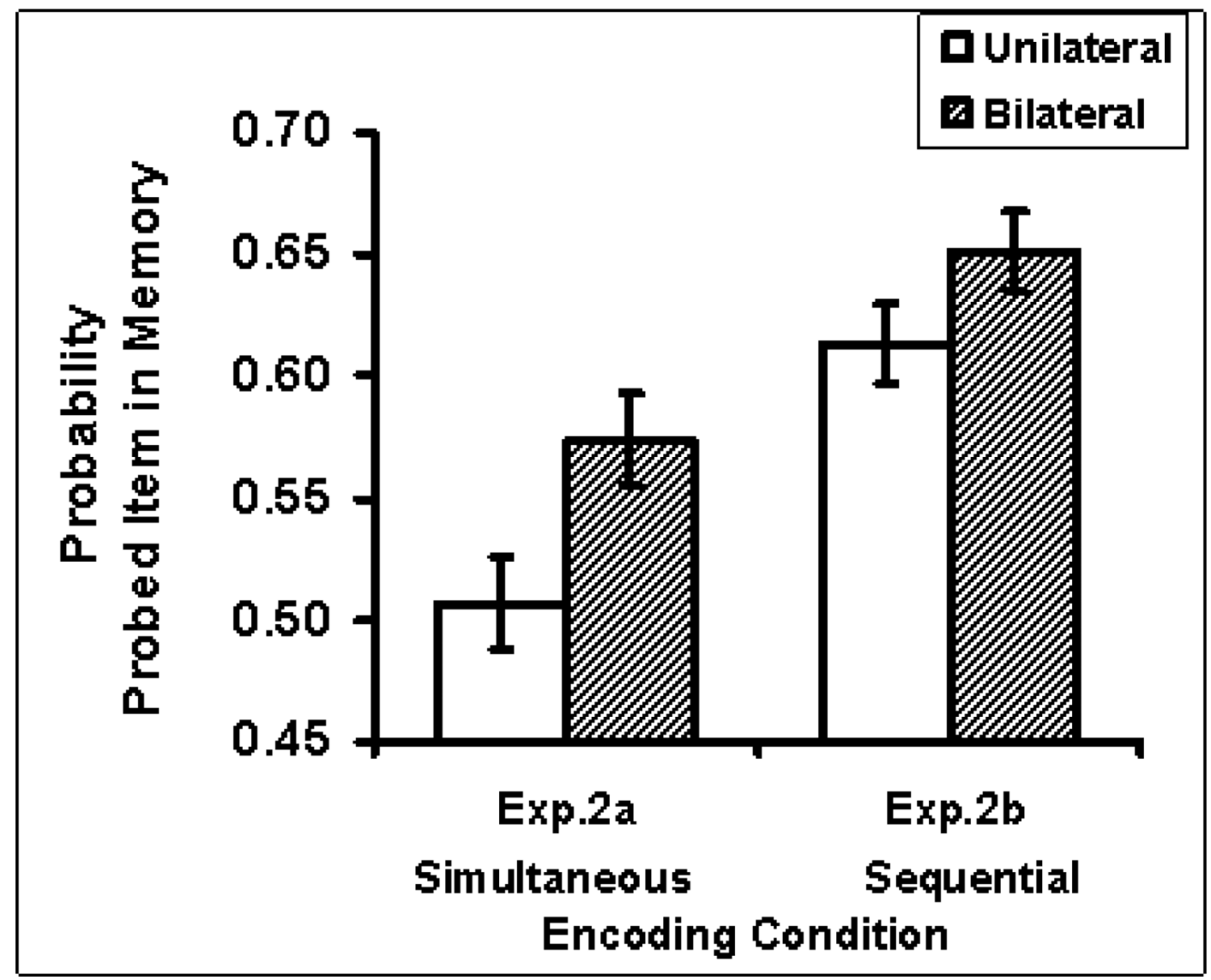

Figure 11.

Probability that the probed items were stored in memory (i.e., Pmem) for the unilaeral and bilateral displays under the simultaneous and sequential encoding processes in Experiment $2 \mathrm{a}$ and $2 \mathrm{~b}$, respectively. The error bar represents $95 \%$ confidence interval (Cousineau, 2005). 


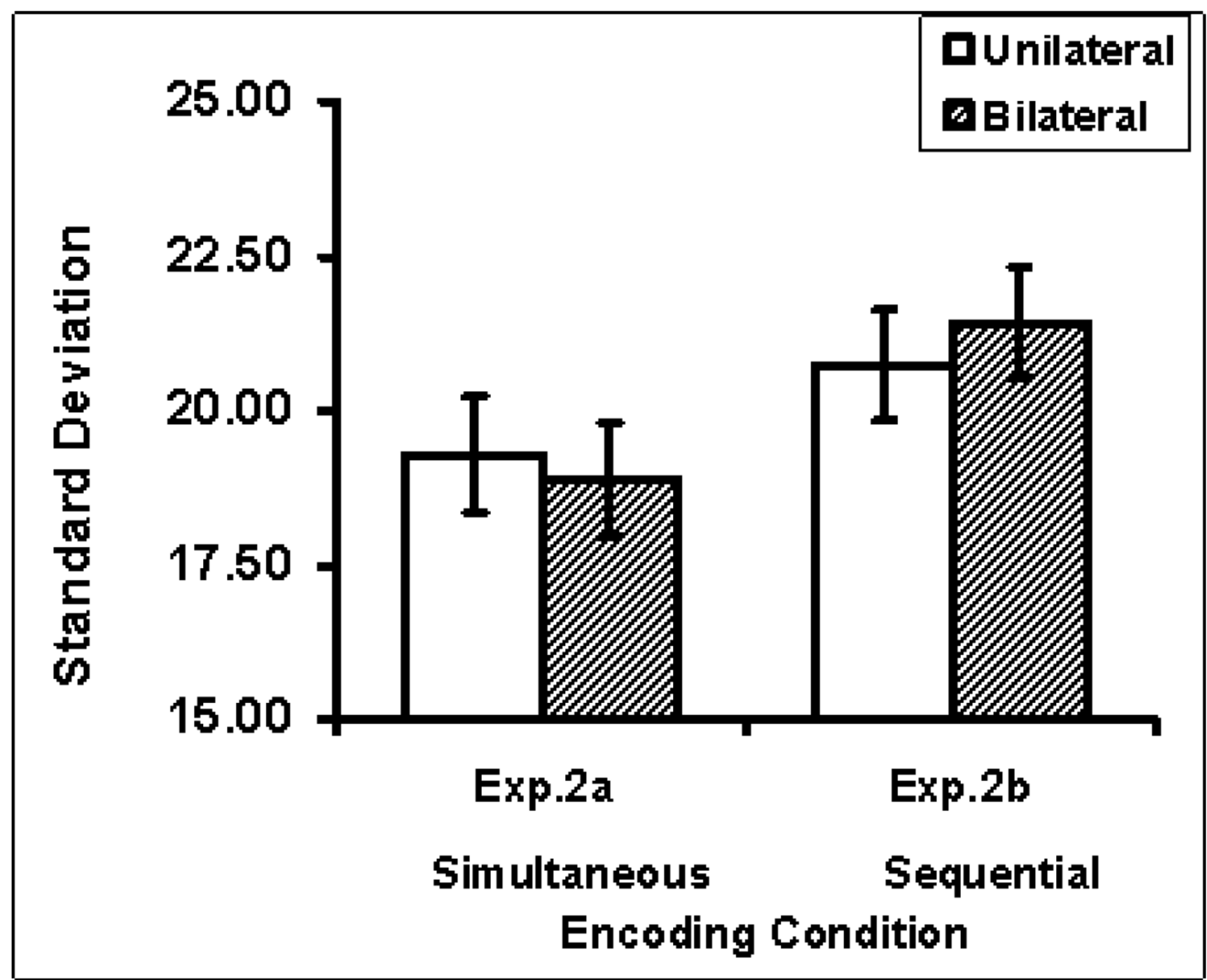

Figure 12.

Precision of the stored representations (i.e., SD) for the unilateral and bilateral displays under the simultaneous and sequential encoding processes in Experiment $2 \mathrm{a}$ and $2 \mathrm{~b}$, respectively. The error bar represents $95 \%$ confidence interval (Cousineau, 2005). 


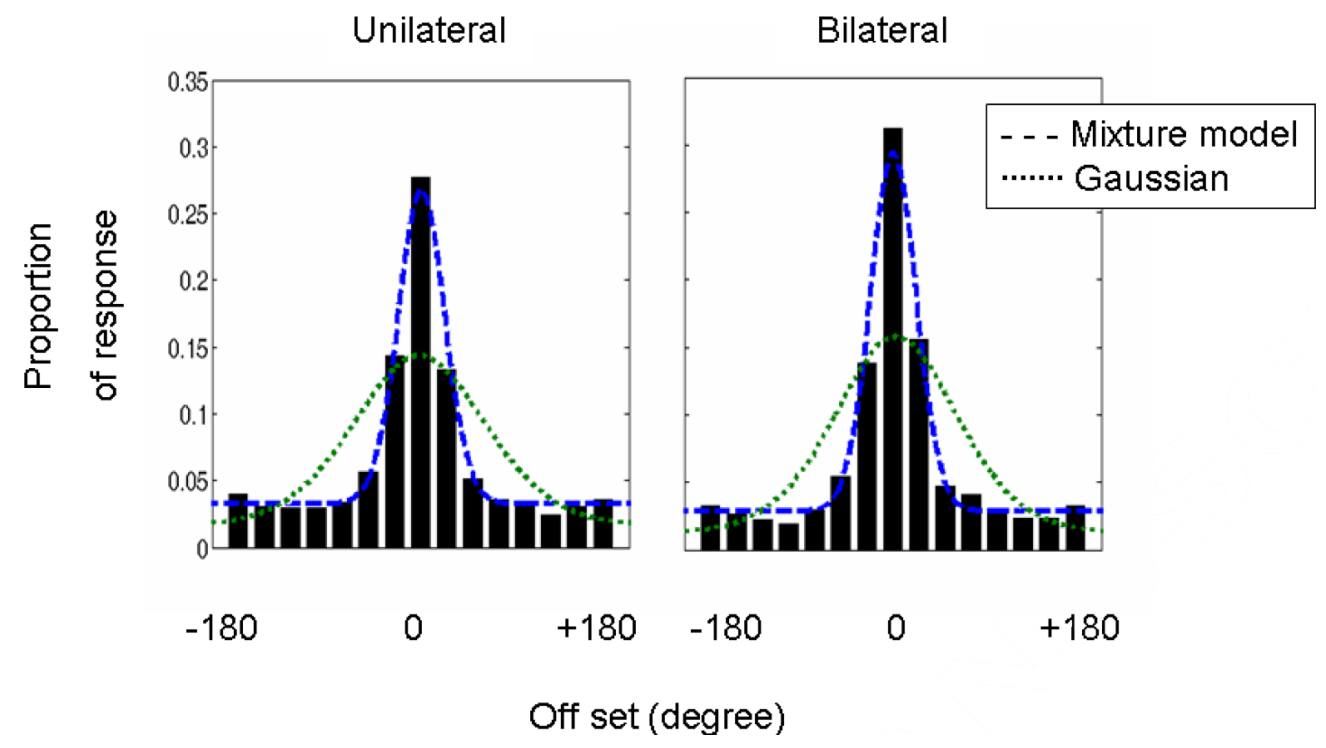

Figure 13.

A distribution across all subjects for recall error for the uni- and bi-lateral displays under the simultaneous encoding processes in Experiment 2a. The two dotted lines correspond to a fit for the mixture model (blue) and Gaussian distribution (green). The proportion of response was calculated by the frequency of response in each bin divided by he total $\mathrm{N}$. 


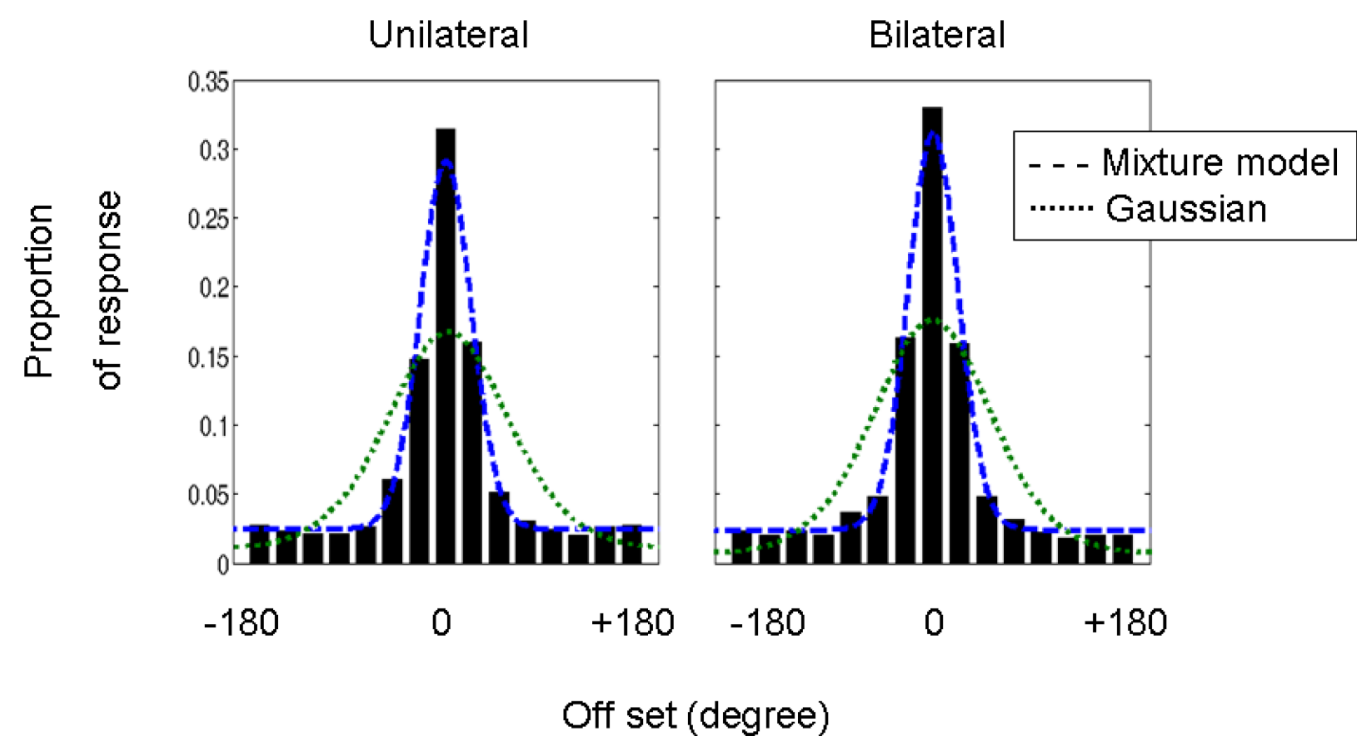

Figure 14.

A distribution across all subjects for recall error for the uni- and bi-lateral displays under the sequential encoding processes in Experiment $2 \mathrm{~b}$. The two dotted lines correspond to a fit for the mixture model (blue) and Gaussian distribution (green). The proporton of response was calculated by the frequency of response in each bin divided by the total $\mathrm{N}$. 